\title{
CAMBIOS ENTÉSICOS EN GUARANÍES PRE-HISPÁNICOS DEL DELTA INFERIOR DEL RÍO PARANÁ
}

\author{
ENTHESEAL CHANGES IN PRE-HISPANIC GUARANÍ FROM THE LOWER \\ PARANA RIVER DELTA
}

\author{
Bárbara Mazza ${ }^{1}$ \\ ${ }^{1}$ Instituto Nacional de Antropología y Pensamiento Latinoamericano
}

PALABRAS CLAVE: horticultores; cuenca del Plata; cazadores-recolectores; entesis

RESUMEN El objetivo de este trabajo es caracterizar los cambios entésicos de la población horticultora (guaraní) del Delta inferior del río Paraná y compararlos con aquellos de los cazadores-recolectores que habitaron la región hacia fines del Holoceno tardío. Para esto se analizó la robusticidad de las entesis de húmeros, fémures y tibias. Los resultados señalan una amplia variabilidad de cambios entésicos entre los guaraníes que pueden corresponderse con la composición sexual y etaria de la muestra, así como con el tamaño corporal y factores mecánicos. Por otro lado, se observó que los individuos recuperados en sitios guaraníes presentan mayores cambios entésicos que los cazadoresrecolectores. Si bien estas diferencias también podrían estar relacionas con factores biológicos y genéticos, es posible que se deban a diferentes adaptaciones mecánicas relacionadas con sus prácticas de subsistencia y equipamientos tecnológicos. Rev Arg Antrop Biol 22(1), 2020. doi: $10.24215 / 18536387 \mathrm{e} 010$

KEYWORDS horticulturists; La Plata basin; hunter-gatherer; enthesis

ABSTRACT The goal of this study is to characterize the entheseal changes of the horticultural population (guaraníes) from the lower Paraná river Delta and to compare them with the entheseal changes from hunter-gatherer societies that inhabited the same region at the end of Late Holocene. To this end, the entheseal robusticity of humerus, femur and tibia was analyzed. The results show a wide variability of entheseal changes among guaraní that could be related to the age and sex composition of the sample, body size and/or mechanical loading. Individuals recovered from guarani's archaeological sites have more pronounced entheseal changes than the hunter-gatherers. Besides biological and genetic factors, it is possible that these differences were caused by different mechanical adaptations according to their subsistence practices and technological equipment. Rev Arg Antrop Biol 22(1), 2020. doi: 10.24215/18536387e 010
Hacia fines del Holoceno tardío, la región que comprende el sector deltaico de la cuenca inferior del río Paraná estuvo habitada por sociedades que practicaban la horticultura. Dadas sus semejanzas con los guaraníes históricos de origen amazónico en su estilo cerámico y prácticas mortuorias, su registro arqueológico fue englobado bajo una unidad arqueológica llamada guaraní (Ambrosetti, 1895; Loponte y Acosta, 2013; Lothrop, 1932; Outes, 1918; Rodríguez, 2008; Torres, 1911; Vignati, 1941). Estas poblaciones tendrían su origen en los bosques septentrionales cálidos y húmedos de Brasil (Noelli, 2008) y habrían descendido por el río Uruguay para llegar al Río de la Plata un poco antes de los 700 años AP (Loponte, Acosta, Capparelli y Pérez, 2011). Allí se encontraron con poblaciones de cazadores-recolectores complejos que ocupaban el área como mínimo desde los 2300 años AP (Loponte, Acosta y Mucciolo, 2012). Ambos grupos poblacionales tuvieron diferentes prácticas de subsistencia, tecnología, patro- nes estilísticos cerámicos, prácticas mortuorias y una distribución diferencial en el espacio (ver más abajo).

Los estudios bioarqueológicos sobre restos guaraníes en el Paraná inferior son escasos y estuvieron concentrados en la caracterización de sus prácticas mortuorias (Ambrosetti, 1895; Lothrop, 1932; Mazza, Acosta y Loponte, 2016; Vignati, 1941). Si bien en Brasil se avanzó sobre otros aspectos, como ser la salud oral (Müller y Mendonça de Souza, 2011) o bien estudios sobre distancias biológicas (Neves, Bernardo,

Financiamiento: Beca postdoctoral del CONICET.

*Correspondencia a: Bárbara Mazza. Instituto Nacional de Antropología y Pensamiento Latinoamericano de Buenos Aires. 3 de febrero 1378. CC1426BJN CABA. Argentina. E-mail: barbara_mazza@yahoo.com.ar

Recibido 8 Noviembre 2018; aceptado 12 Abril 2019

doi:10.24215/18536387e010 
Okumura, Ferreira de Almeida y Strauss, 2011), el estado fragmentario de los restos óseos no es el propicio para estudiar variaciones en la morfología ósea postcraneal (Müller y Mendonça de Souza, 2011; Prous, 2011). Al contrario, el registro bioarqueológico de la población guaraní del sector inferior del río Paraná presenta un buen estado de preservación, lo cual permite estudiar diversos aspectos biológicos y culturales relacionados con variaciones en el tamaño y forma de los elementos óseos del postcráneo en su límite más austral de distribución espacial.

El objetivo de este trabajo es caracterizar los cambios entésicos de la población horticultora (guaraní) del Delta inferior del río Paraná que habitó la región hacia fines del Holoceno tardío. Los cambios entésicos comprenden modificaciones en la morfología de las entesis (i.e. lugares de inserción de ligamentos, músculos y tendones) (Villotte et al., 2016) como resultado de la acción sinérgica de factores etarios, hormonales, mecánicos y genéticos. Si bien hace un tiempo se pensaba que había una relación directa entre los cambios entésicos y el estrés mecánico, recientes investigaciones también demostraron que son más comunes entre individuos mayores a los 40-50 años y masculinos así como entre aquellos de gran tamaño corporal (cf. Jurmain, Alves Cardoso, Henderson y Villote, 2012). Este trabajo se centra en el análisis de una muestra de húmeros, tibias y fémures de individuos adultos a la vez que explora su relación con variables biológicas y culturales. Este estudio nos permitirá acceder a aspectos sociales y biológicos de esta sociedad no estudiados previamente.

La introducción de la agricultura o bien la intensificación de los cultivos produjo cambios en la morfología ósea de sociedades pasadas. Algunas investigaciones señalan que hubo una reducción general de la robusticidad postcraneal desde las sociedades cazadoras-recolectoras a las agricultoras, debido a cambios tecnológicos y en los patrones de movilidad (Henderson, 2013a; Larsen, 1995; Ruff, 1987). No obstante, hay casos particulares donde aparece el patrón contrario. Es decir en algunas sociedades el traspaso hacia la agricultura o hacia una horticultura intensiva produjo un aumento en el estrés mecánico incrementando la robusticidad ósea de las diáfisis (Bridges, Blitz y Solano, 2000) o bien de las entesis (Chapman, 1997; Eshed, Gopher, Galili y Hershkovitz, 2004; Shuler, Zeng y Danforth, 2012). Esta contraposición de patrones morfológicos es probable que se deba a distintas prácticas culturales así como a diferencias en la composición sexual y etaria del grupo, su ambiente y estado de conservación de las muestras (Henderson, 2013a; Larsen, 1995; Ruff, 1987). A pesar de estas discrepancias y sus razones subyacentes puede sostenerse que las sociedades cuyo sustento económico proviene fundamentalmente de los cultivos presentan una morfología ósea diferente a aquellas con subsistencia principalmente cazadora-recolectora. En base a esto, en este trabajo también nos proponemos explorar las diferencias o similitudes en los cambios entésicos entre el grupo guaraní y los cazadores-recolectores del área y sus posibles causas. Dado que ambos grupos difieren en sus prácticas de subsistencia esperamos que también presenten diferencias en la morfología de sus entesis.

\section{El registro arqueológico de sociedades guaraníes y cazadoras-recolectoras del Delta inferior del río Paraná}

Los sitios arqueológicos guaraníes de la región del humedal del Paraná inferior se encuentran en el sector insular (comúnmente llamado Delta), mientras que los sitios cazadores-recolectores están distribuidos por toda la región, tanto en el sector continental como en el sector insular. La circunscripción espacial al sector deltaico del grupo guaraní fue explicada como el resultado de su llegada tardía a la región, donde ya existía una significativa saturación del espacio por parte de las poblaciones cazadorasrecolectoras locales (Loponte et al., 2011).

La población guaraní del Delta inferior del río Paraná fue caracterizada etnográfica y arqueológicamente, entre otros aspectos, por la horticultura de maíz y calabazas, la caza de mamíferos de mediano y gran porte (Blastocerus dichotomus, Hydrochaeris hydrochaeris, Myocastor coypus) y la pesca (Acosta, Loponte y Mucciolo, 2010a, 2010b; Métraux, 1948). A su vez, su cerámica tiene un estilo peculiar, definida por el corrugado y la ejecución de motivos geométricos pintados en rojo y/o negro sobre un fondo blanco. La tipología de la cerámica inclu- 
ye grandes recipientes, a menudo con carenas y bordes reforzados, absolutamente originales y diferentes al resto del registro arqueológico de la región (Loponte et al., 2011; Lothrop, 1932; Outes, 1918). Las prácticas funerarias incluían la inhumación primaria y secundaria, a menudo en grandes vasijas que también constituyó una practica distintiva de estos grupos (Lothrop, 1932; Mazza et al., 2016; Vignati, 1941). Su sistema de armas incluía el uso del arco y flecha, boleadoras y anzuelos. Es posible que emplearan una gran cantidad de artefactos de madera que no han perdurado en el registro arqueológico local. También son frecuentes las hachas pulidas petaliformes o rectas utilizadas para la tala del bosque para la preparación de las áreas de cultivo mediante roza y quema (Acosta et al., 2010b; Loponte y Acosta, 20032005; Lothrop, 1932; Métraux, 1948; Outes, 1918; Pérez, Silvestre y Buc, 2019).

En cambio, las sociedades cazadoras-recolectoras del río Paraná inferior tenían una dieta basada principalmente en la pesca, junto al consumo de mamíferos (Blastocerus dichotomus, Ozotoceros bezoarticus, Cavia aperea, Myocastor coypus) y de vegetales (e.g. Oryza latifolia, Cucurbita sp., Syagrus romanzzofiana), que habrían sido recolectados en estado silvestre o bien cultivados a pequeña escala (Acosta et al., 2010a; Acosta y Ríos Román, 2013; Loponte, Acosta y Corriale, 2016). Su cerámica era predominantemente lisa o incisa, a veces con apéndices zoomorfos y formas tubulares (Loponte y Acosta, 2016; Pérez y Cañardo, 2004), y sus entierros eran colocados directamente en contacto con la tierra (Mazza y Loponte, 2012). Su tecnología está compuesta por un amplio repertorio de instrumentales óseos y líticos, los cuales comprenden el uso de arco y flecha, propulsores, boleadoras y arpones (Sacur Silvestre, Buc, Acosta y Loponte, 2013). A diferencia de los guaraníes, no se han registrado hachas de mano ni anzuelos en los sitios cazadores-recolectores (Buc y Loponte, 2007; Loponte et al., 2011)

\section{MATERIAL Y MÉTODOS}

La muestra de la unidad arqueológica guaraní proviene de tres sitios con fechados radiocarbónicos de 700-400 años AP (Fig. 1): Arroyo Fredes (Loponte y Acosta, 2003-2005; Vignati,
1941), Arroyo La Glorieta (Mazza et al., 2016) y Arroyo Malo (Lothrop, 1932). El total de elementos óseos analizados es de 79, de los cuales 22 son húmeros, 38 son fémures y 19 son tibias, correspondientes a un mínimo número de 22 individuos adultos (Tabla 1). Los cúbitos y radios fueron excluidos del análisis por su reducido número ( $\mathrm{n}=7 \mathrm{y} \mathrm{n}=6$, respectivamente). A su vez, debido a que las lateralidades derecha e izquierda están representadas por un relativamente bajo número de elementos óseos, se tomó la decisión de no distinguir por lateralidad para, de esta manera, incrementar el tamaño de la muestra y así facilitar los análisis estadísticos.

Si bien los restos óseos de individuos guaraníes presentan un buen estado de preservación, se tratan de elementos óseos aislados. Esto se debe a que provienen de entierros, en su mayoría seguramente de carácter secundario, en urnas funerarias que fueron recuperados en excavaciones a principios del siglo XX por investigadores del Museo de La Plata, institución donde hoy en día se encuentran resguardados (Mazza et al., 2016; Vignati, 1941). Ambas circunstancias complican la asignación sexual y etaria de los elementos óseos. Si bien en las urnas fueron inhumados individuos de ambos sexos y de diferentes edades (Mazza et al., 2016; Vignati, 1941), estas variables no se pudieron estimar en los huesos largos incluidos en este trabajo. A su vez, los elementos óseos se guardaron agrupados por unidad anatómica, lo cual nos impide establecer algún grado de conexión entre ellos. Por lo tanto, sólo trabajaremos con la categoría de adulto, determinada a partir de la fusión completa de las epífisis (Scheuer y Black, 2000). Por otro lado, a este carácter de desarticulación ósea se suma la falta de datos métricos para esta población que nos permita estimar el sexo de los huesos largos con cierto grado de confianza.

El conjunto correspondiente a la unidad arqueológica cazadora-recolectora (de aquí en más c-r) comprende 10 sitios arqueológicos ubicados cronológicamente entre los 2000-500 años ${ }^{14} \mathrm{C}$ AP (Fig. 1), Arroyo Sarandí (Loponte, 2008; Lothrop, 1932), Garín, La Bellaca sitio 1 (Loponte, 2008), El Cerrillo (Lothrop, 1932), Túmulo II del Brazo Largo (Mazza, 2015; Politis et al., 2017), Arroyo Marieta (Mazza, 2015), Los Talas (Vignati, 1960), Paraná Ibicuy 1/La Argentina (Caggiano, Flores, Méndez y 


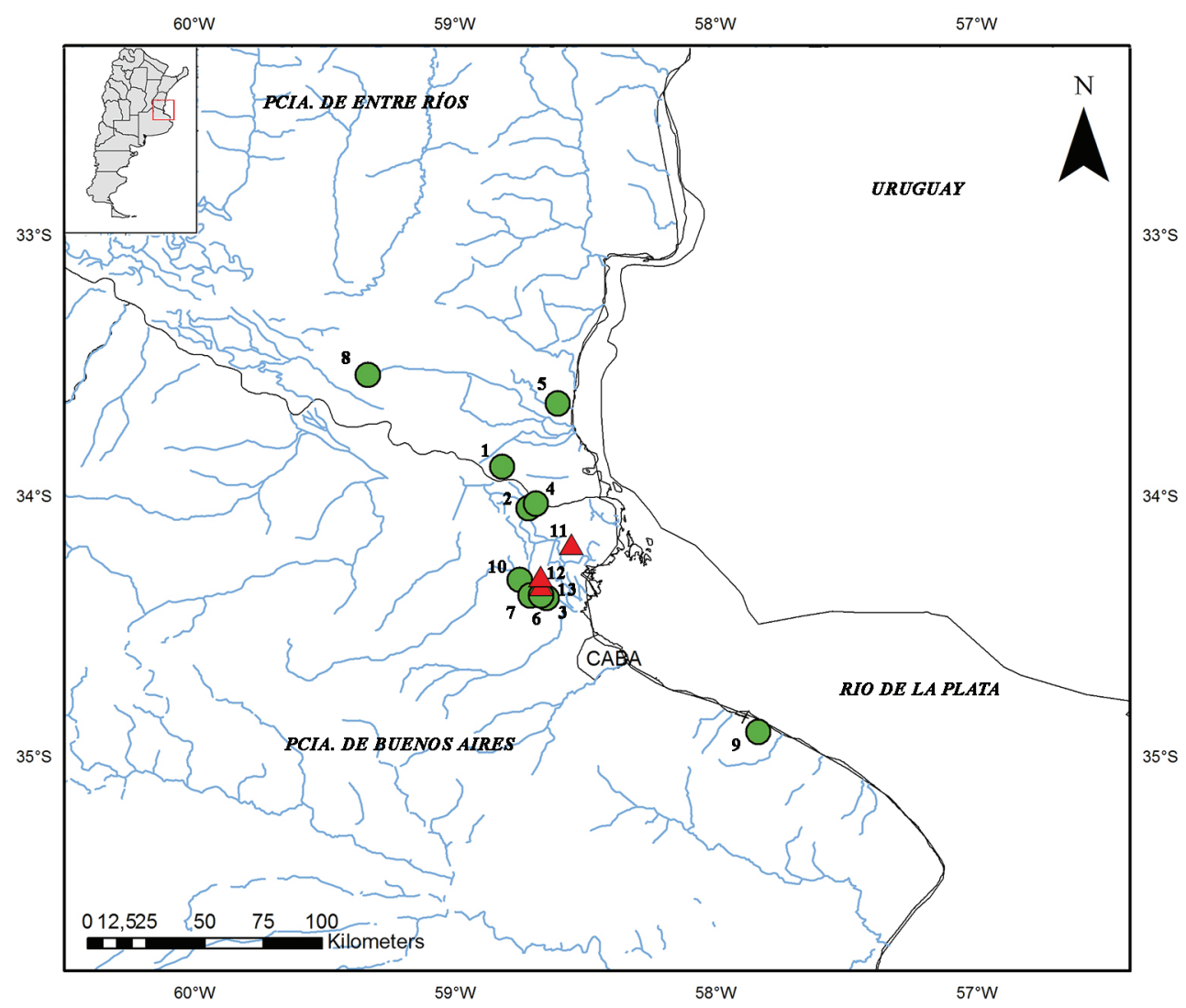

Fig. 1. Ubicación de los sitios arqueológicos guaraníes (triángulos) y cazadores-recolectores (círculos). 1:Túmulo II del Brazo Largo; 2:Arroyo Marieta; 3:Arroyo Sarandí: 4:El Cerrillo; 5:Cerro Lutz; 6:El Cazador sitio 3; 7:Garín; 8:Paraná Ibicuy 1/La Argentina; 9:Los Talas; 10:La Bellaca sitio 1; 11:Arroyo Fredes; 12:Arroyo Malo; 13:Arroyo La Glorieta.

TABLA 1. Descripción de la muestra

\begin{tabular}{cccccccc}
\hline & \multicolumn{2}{c}{ Húmero } & \multicolumn{2}{c}{ Fémur } & \multicolumn{2}{c}{ Tibia } & \multirow{2}{*}{ MNI $^{\mathrm{a}}$} \\
& $\mathrm{I}$ & $\mathrm{D}$ & $\mathrm{I}$ & $\mathrm{D}$ & $\mathrm{I}$ & $\mathrm{D}$ & \\
\hline Arroyo Fredes & 9 & 10 & 13 & 17 & 8 & 9 & 17 \\
Arroyo La Glorieta & 1 & 1 & 2 & 3 & 0 & 0 & 3 \\
Arroyo Malo & 0 & 1 & 1 & 2 & 1 & 1 & 2 \\
$\begin{array}{c}\text { Total de elementos óseos } \\
\text { analizados }\end{array}$ & 10 & 12 & 16 & 22 & 9 & 10 & 22 \\
\hline
\end{tabular}

I:izquierdo; D:derecho; MNIa: estimación del número de individuos en base al número máximo de elementos anatómicos derechos o izquierdos (White, 1953).

Salceda, 1978), El Cazador sitio 3 (Loponte et al., 2016) y Cerro Lutz (Mazza, 2010). La muestra se compone de 230 huesos largos (69 húmeros, 86 fémures y 75 tibias), pertenecientes a 73 individuos adultos de ambos sexos (50 masculinos y 23 femeninos). Los cambios entésicos de esta muestra ya fueron analizados en otro trabajo (Mazza, 2018) y sólo serán retomados en esta oportunidad con fines comparativos.

Para estudiar la influencia del tamaño corporal en los cambios entésicos se construyeron variables compuestas para cada hueso largo por separado a partir del promedio de los valoresz de la longitud máxima y tamaño de ambas 
TABLA 2. Estadística descriptiva de las variables usadas para la construcción del valor-z (proxy del tamaño corporal) en la muestra del grupo guaraní

\begin{tabular}{lcccc}
\hline & Media & De & Min & Máx \\
\hline Húmero (n=22) & & & & \\
Long máx & 311.18 & 14.01 & 278.00 & 330.00 \\
Ep px & 61.94 & 5.48 & 53.11 & 72.08 \\
Ep ds & 46.97 & 4.02 & 38.58 & 53.13 \\
valor-z & 0 & 0.94 & -2.02 & 1.56 \\
& & & & \\
Fémur (n=38) & & & 372.00 & 477.00 \\
Long máx & 429.32 & 28.81 & 36.09 & 50.3 \\
Ep px & 44.47 & 3.62 & 63.00 & 90.00 \\
Ep ds & 76.26 & 7.13 & -1.89 & 1.52 \\
valor-z & 0.00 & 0.90 & & \\
& & & & 401.00 \\
Tibia (n=19) & & & 312.00 & 86.00 \\
Long máx & 361.89 & 23.93 & 57.00 & 56.00 \\
Ep px & 72.42 & 7.60 & 39.00 & 1.61 \\
Ep ds & 48.53 & 5.31 & -1.84 & \\
valor-z & 0.00 & 0.94 & & \\
\hline
\end{tabular}

De:desviación estándar; Min:valor mínino; Máx:valor máximo; Long máx:longitud máxima; Ep px:epífisis proximal; Ep ds:epífisis distal

epífisis (Tabla 2). Los valores-z son unidades de medida estadística estandarizadas. Es decir, informan a cuántos desvíos estándar se encuentra el valor de una observación o medición con respecto a la media. Los valores pueden ser negativos o positivos dependiendo si se ubican por debajo o por encima de la media. Se calcula a partir de la sustracción de la media poblacional (o de la muestra) del valor observado, dividido por la desviación estándar. Las medidas de los huesos largos fueron tomadas con una tabla osteométrica y calibre digital (Buikstra y Ubelaker, 1994). No se encontraron diferencias significativas entre dos instancias de medición realizadas con una diferencia de 15 días entre sí para evaluar la presencia de errores intraobservador (ANOVA de medidas repetidas $p<0,010$, Índice de correlación intraclase $<0,60$ ).

A nivel mundial las mujeres suelen presentar tamaños corporales más pequeños que los hombres (Frayer y Wolpoff, 1985), correlación que ha sido significativa en otros trabajos (Weiss, 2003). Por lo tanto, los valores-z también se utilizaron como proxy del sexo de los individuos en la muestra del grupo guaraní. Si bien no co- nocemos el grado de dimorfismo sexual de esta población ni las medidas que corresponden a cada sexo, tomaremos a los valores-z negativos (es decir aquellos que se encuentran por debajo de la media) como representantes de posibles femeninos, y los valores-z positivos como posibles masculinos.

Se analizaron en total nueve entesis que, de acuerdo a su composición tisular y ubicación en el elemento óseo, se clasifican en fibrocartilaginosas (braquiorradial, iliopsoas y cuádriceps) y fibrosas (deltoides, dorsal ancho, pectoral mayor, glúteo mayor, vasto medial y sóleo) (Benjamin et al., 2002). Para el registro de los cambios entésicos se acudió a la metodología desarrollada por Mariotti, Facchini y Belcastro (2007). Este método analiza la robusticidad de las entesis de acuerdo a una escala ordinal de expresión que se traduce en $1=$ robusticidad débil a moderada, $2=$ robusticidad alta, $3=$ robusticidad muy alta. Si bien la designación de estos gradientes puede parecer subjetiva, el método cuenta con una buena descripción y fotografías de cada grado de robusticidad para cada elemento óseo lo cual permite un buen grado de dife- 
renciación. Para este método, tampoco se observaron errores intraobservador significativos, calculados a partir de dos instancias de registro con una diferencia de 15 días $(78-100 \%$ de acuerdo; índice de concordancia Kappa > 0,60; coeficiente de Krippendorff 0,66-0,88).

Recientemente se desarrolló un nuevo método para el estudio de los cambios entésicos, el Método Coimbra (Henderson, Mariotti, Pany-Kucera, Villote, y Wilczak, 2013, 2016). Sin embargo, no se aplicó en este trabajo debido a dos razones. La primera es que la muestra de cazadores-recolectores con la cual se compararon los cambios entésicos del grupo guaraní fue analizada previamente con el método de Mariotti et al. (2007). En segundo lugar, el Método Coimbra solo es aplicable a entesis fibrocartilaginosas; es decir, que no comprende inserciones musculares grandes y fuertes como son el pectoral mayor y el deltoides (Benjamin et al., 2002). Esta exclusión se debe a que se desconoce el estado inalterado de las entesis fibrosas (Villote et al., 2016). Sin embargo, los grados de robusticidad del método de Mariotti et al. (2007) permiten distinguir estadios gráciles $\mathrm{y}$ robustos, considerados suficientes para hacer comparaciones.

Se excluyeron del análisis a los individuos que presentaran algún tipo de patología, trauma o crecimiento óseo anormal en el esqueleto en general o bien en el elemento óseo en particular (Henderson, 2013b; Resnick y Niwayama, 1983). Es importante destacar que no se detectaron entesis que presentaran erosiones corticales en extensión o crecimiento óseo en exceso (Henderson, 2013a; Resnick y Niwayama, 1983).

\section{Análisis de los cambios entésicos}

La caracterización de la morfología de las entesis del grupo guaraní se realizó a través del cálculo de las frecuencias relativas de los grados de robusticidad para cada entesis. Luego, para evaluar la influencia del tamaño corporal y/o del sexo en los cambios entésicos, se realizaron correlaciones de Spearman entre los grados de robusticidad y los valores-z de cada elemento anatómico. Por otro lado, dado que los músculos actúan sinérgicamente también se realizaron correlaciones de Spearman entre los grados de robusticidad de las distintas entesis. Este último análisis también se realizó en la muestra de c-r.

Para comparar los cambios entésicos de la muestra guaraní con aquella de los c-r se generó un modelo lineal generalizado (MLG) (McCullagh y Nelder, 1989). Este estadístico presenta la posibilidad de analizar una serie de datos con cualquier tipo de distribución, para evaluar el impacto de $p$ variables explicativas (i.e. variables independientes) sobre una variable de respuesta (variable dependiente). En este trabajo, la variable dependiente son los cambios entésicos y las variables independientes son las unidades arqueológicas (variable categórica, codificada como $1=$ guaraní; $2=\mathrm{c}-\mathrm{r}$ ) y el tamaño corporal (valores-z, variable continua). Los valores de los parámetros generados por el modelo $(B)$ indican la fuerza y asociación de la variable explicativa con la dependiente. De esta forma, por ejemplo, un valor- $B$ positivo en la unidad arqueológica señala que el grupo guaraní tiene más cambios entésicos que los c-r.

La matriz de covarianza usada en el MLG corresponde al estimador robusto, ya que provee una buena estimación de la covarianza (Chrisletta y Spini, 2004). Dado que la variable dependiente es ordinal, el modelo utilizado fue el de respuesta ordinal con función de enlace logit acumulado y efectos principales con análisis tipo III. Este modelo evalúa la contribución (efecto) de cada variable explicativa mientras controla el efecto de las restantes. Es decir, en este caso, analiza si hay diferencias entre los c-r y guaraníes a medida que controla el tamaño corporal de ambos grupos.

Cuando se comparan varias variables aumenta la probabilidad de rechazar erróneamente la hipótesis nula; por lo tanto, los valores $p$ fueron recalculados usando la corrección de Holm-Bonferroni para comparaciones múltiples (Nikita, 2017). Todos los análisis estadísticos se realizaron con el programa IBM SPSS Statistics v. 19 (IBM Corp., 2010) con un $\alpha=0,05$.

\section{RESULTADOS}

En la Tabla 3 se muestra la distribución de los grados de robusticidad por entesis para los tres sitios arqueológicos guaraní. Se encontraron diferencias estadísticamente significativas entre ellos en los grados de robusticidad del glúteo mayor y del dorsal ancho. Sin embargo, 


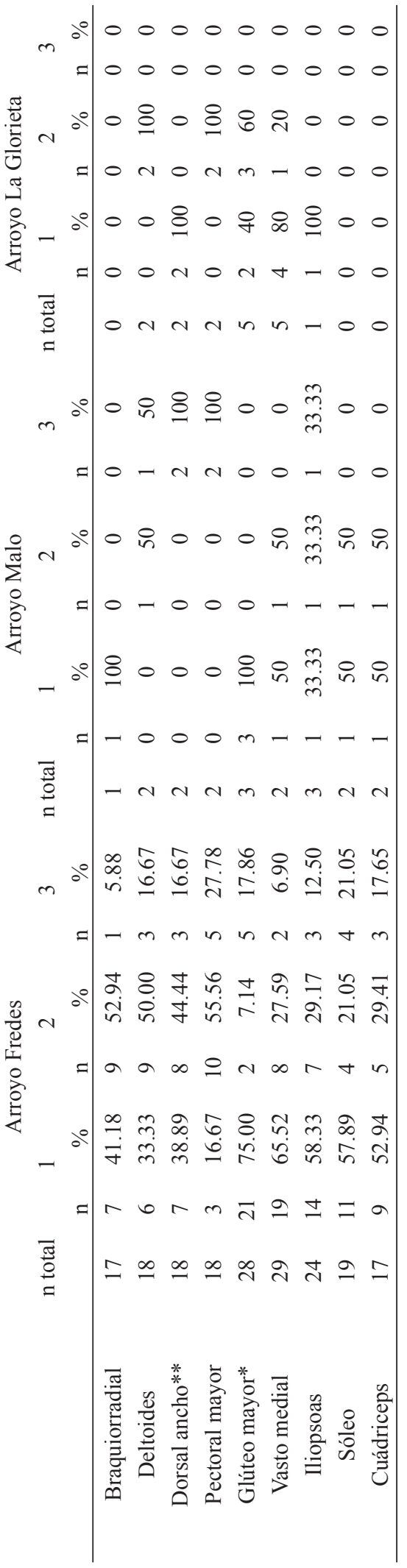

estas diferencias seguramente se deban al tamaño de muestra de cada sitio arqueológico o bien a diferencias en su composición sexual y/o etaria. Como puede verse en la Tabla 3, la cantidad de entesis analizadas oscila entre una y tres para Arroyo Malo, y entre una y cinco en Arroyo La Glorieta, careciendo de registros para algunas entesis en este último sitio arqueológico. Si bien ambas diferencias estadísticas deben revaluarse en muestras más grandes, para este trabajo analizaremos la muestra como un todo a los fines de no descartar las entesis de los sitios arqueológicos con muestras pequeñas.

Las entesis de los miembros inferiores del grupo guaraní se caracterizan por presentar una robusticidad leve; en cambio las de los miembros superiores se distribuyen, principalmente, entre los grados leves y moderados (Fig. 2). Al comparar estas distribuciones con las del grupo c-r, se observa que los guaraníes tienen mayores frecuencias en los grados de robusticidad moderado y alto en las entesis de los miembros superiores. En cambio, no parece haber diferencias en las entesis de los miembros inferiores, con excepción del iliopsoas, donde los c-r muestran una mayor frecuencia en el grado moderado. Los resultados del MLG corroboran algunas de estas diferencias (Tabla 4). El braquiorradial, dorsal ancho, pectoral mayor y vasto medial presentan mayores cambios entésicos entre los guaraníes.

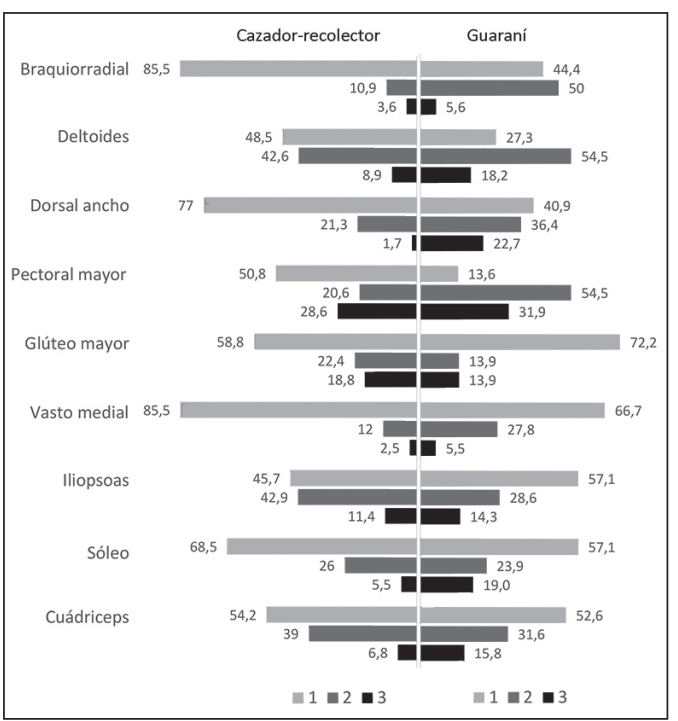

Fig. 2. Frecuencias relativas de los grados de robusticidad para cada entesis del grupo guaraní y cazador-recolector. 
TABLA 4. Resultados del modelo lineal generalizado

\begin{tabular}{ccccccc}
\hline Entesis & & AICc & \multicolumn{2}{c}{ Unidad arqueológica } & \multicolumn{2}{c}{ valor-z } \\
& $\mathrm{n}$ & & $\mathrm{B}$ & $\mathrm{p}$ & $\mathrm{B}$ & $\mathrm{p}$ \\
\hline Braquiorradial & 73 & 88.99 & 1.935 & $\mathbf{0 . 0 0 2 *}$ & 0.485 & 0.191 \\
Deltoides & 90 & 158.96 & 1.005 & $\mathbf{0 . 0 3 4}$ & 0.463 & 0.054 \\
Dorsal ancho & 83 & 125.82 & 1.770 & $\mathbf{0 , 0 0 1 *}$ & 0.142 & 0.566 \\
Pectoral mayor & 85 & 170.32 & 0.912 & $\mathbf{0 . 0 1 4} *$ & 0.331 & 0.154 \\
Glúteo mayor & 121 & 199.05 & -0.095 & 0.832 & 1.065 & $<\mathbf{0 . 0 0 1 *}$ \\
Vasto medial & 119 & 132.94 & 1.283 & $\mathbf{0 . 0 2 2} *$ & 0.372 & 0.249 \\
Iliopsoas & 98 & 178.29 & 0.277 & 0.589 & 0.958 & $<\mathbf{0 . 0 0 1 *}$ \\
Sóleo & 85 & 138.54 & 1.116 & 0.062 & 0.681 & $\mathbf{0 . 0 1 7 *}$ \\
Cuádriceps & 78 & 121.07 & 0.827 & 0.164 & 1.338 & $<\mathbf{0 . 0 0 1 *}$ \\
\hline
\end{tabular}

AICc: Criterio de información de Akaike corregido.A menor valor, mejor calidad del modelo; B: valor del parámetro; *: Permanece significativo luego de la corrección de Holm-Bonferroni. Resaltado en negrita los valores estadísticamente significativos.

Es posible que la mayor robusticidad de las entesis entre los guaraníes se deba a que esta muestra contenga un mayor número de individuos masculinos que la de los c-r o, simplemente, que esté conformada únicamente por individuos de dicho sexo. Esto se debe a que los masculinos suelen presentar mayores cambios entésicos que los femeninos (inclusive en la muestra de c-r del Paraná inferior). Por lo tanto, se decidió realizar un nuevo análisis de MLG eliminando a los individuos femeninos de la muestra c-r (Tabla 5). Estos nuevos resultados coinciden en su mayoría con los obtenidos previamente, es decir que los guaraníes continúan mostrando mayores cambios entésicos que los c-r en las mismas entesis que el modelo anterior, con excepción del pectoral mayor que ya no muestra resultados significativos.

Los resultados de ambos MLG también mostraron que los tamaños corporales más grandes de ambos grupos tienen mayores cambios entésicos en los miembros inferiores. Esta correlación positiva también se detectó al interior de los guaraníes en todas las entesis de los miem-

TABLA 5. Modelo lineal generalizado sin individuos femeninos del grupo cazador-recolector

\begin{tabular}{ccccccc}
\hline Entesis & & AICc & \multicolumn{2}{c}{ Unidad arqueológica } & \multicolumn{2}{c}{ valor-z } \\
& $\mathrm{n}$ & & $\mathrm{B}$ & $\mathrm{p}$ & $\mathrm{B}$ & $\mathrm{p}$ \\
\hline Braquiorradial & 60 & 75.99 & $\underline{2.272}$ & $\underline{\mathbf{0 . 0 0 1 *}}$ & 0.803 & 0.097 \\
Deltoides & 76 & 140.96 & 1.077 & $\mathbf{0 . 0 4 6}$ & 0.550 & 0.113 \\
Dorsal ancho & 70 & 117.31 & $\underline{1.456}$ & $\mathbf{0 . 0 0 6}$ & -0.218 & 0.545 \\
Pectoral mayor & 72 & 149.85 & 0.842 & 0.051 & 0.253 & 0.433 \\
Glúteo mayor & 101 & 182.62 & -0.270 & 0.594 & $\underline{0.905}$ & $\underline{\mathbf{0 . 0 0 4}}$ \\
Vasto medial & 99 & 107.70 & $\underline{2.096}$ & $\underline{\mathbf{0 . 0 0 2}}$ & 1.081 & $\mathbf{0 . 0 0 1 *}$ \\
Iliopsoas & 80 & 153.06 & -0.020 & 0.976 & $\underline{0.764}$ & $\mathbf{0 . 0 2 5}$ \\
Sóleo & 74 & 126.25 & 1.246 & 0.050 & $\underline{0.818}$ & $\underline{\mathbf{0 . 0 0 9 *}}$ \\
Cuádriceps & 63 & 114.80 & 0.761 & 0.218 & $\underline{1.293}$ & $\underline{\mathbf{0 . 0 0 3}}$ \\
\hline
\end{tabular}

AICc: Criterio de información de Akaike corregido. A menor valor, mejor calidad del modelo; B: valor del parámetro; *: Permanece significativo luego de la corrección de Holm-Bonferroni. Resaltado en negrita los valores estadísticamente significativos. Subrayado los valores que coinciden con los del modelo linear generalizado de la Tabla 4. 
bros inferiores (con excepción del sóleo) y en el pectoral mayor (Tabla 6). A su vez, si tomamos como válida la correlación entre tamaño corporal y sexo, estas asociaciones también podrían indicar que los individuos masculinos son los

TABLA 6. Correlación de Spearman entre los cambios entésicos y los valores-z como proxy del tamaño corporal en el grupo guaraní

\begin{tabular}{cccc}
\hline & $\mathrm{n}$ & $\mathrm{rs}$ & $\mathrm{p}$ \\
\hline Braquiorradial & 18 & 0.29 & 0.239 \\
Deltoides & 22 & 0.32 & 0.151 \\
Dorsal ancho & 22 & -0.14 & 0.534 \\
Pectoral mayor & 22 & $\mathbf{0 . 4 7}$ & $\mathbf{0 . 0 2 8}$ \\
Glúteo mayor & 36 & $\mathbf{0 . 5 6}$ & $<\mathbf{0 . 0 0 1}$ \\
Vasto medial & 36 & $\mathbf{0 . 4 2}$ & $\mathbf{0 . 0 1 0}$ \\
Iliopsoas & 28 & $\mathbf{0 . 5 2}$ & $\mathbf{0 . 0 0 4}$ \\
Sóleo & 21 & 0.18 & 0.435 \\
Cuádriceps & 19 & $\mathbf{0 . 4 9}$ & $\mathbf{0 . 0 3 4}$ \\
\hline
\end{tabular}

Resaltado en negrita los valores estadísticamente significativos.

que presentan mayores cambios entésicos.

También se hallaron correlaciones positivas significativas entre algunas entesis; es decir que los valores elevados de robusticidad de una entesis se relacionan con valores elevados de otra entesis (Tabla 7). Entre los guaraníes, los cambios entésicos del deltoides se correlacionan con los del pectoral mayor, los del vasto medial con los del iliopsoas y los del sóleo con los del cuádriceps. Este patrón difiere del observado entre los c-r, ya que en este grupo todas las entesis del húmero y fémur se correlacionan entre sí, con excepción del braquiorradial que, al igual que entre los guaraníes, no se correlaciona con ninguna entesis. A su vez, a diferencia de los guaraníes, no hay correlaciones entre las entesis de la tibia.

\section{DISCUSIÓN}

Los restos óseos de la unidad arqueológica guaraní del sur de la cuenca del Plata presentan cambios entésicos leves, moderados y altos en todas las entesis analizadas. Dado el carácter multifactorial de los cambios entésicos, esta variabilidad podría estar relacionada con la composición sexual y etaria, variaciones en el tamaño corporal, influencia del estrés mecánico y/o sesgo en la muestra.

La edad es uno de los principales factores que intervienen en la morfología de las entesis. En este sentido los individuos de edades más avanzadas suelen tener más cambios entésicos que los más jóvenes (e.g. Alves Cardoso y Henderson, 2010; Milella, Giovanna Belcastro, Zollikofer y Mariotti, 2012; Weiss, Corona y Schultz, 2012). Lamentablemente, las costumbres funerarias de las sociedades guaraníes y las prácticas de los museos nacionales, imponen limitaciones para analizar diferencias en los cambios entésicos entre distintos grupos etarios. Por lo tanto, en este trabajo no podemos saber si los restos analizados pertenecen a individuos adultos de diferentes edades. Sin embargo, dados los antecedentes en la temática, es importante considerar que la edad podría estar influyendo en la variabilidad de cambios entésicos dentro de la muestra analizada. De esta forma, los valores elevados de robusticidad podrían corresponder a individuos de edades avanzadas; lo contrario para los cambios entésicos leves.

Otro factor que está influyendo en los cambios entésicos del grupo guaraní es el tamaño corporal; las unidades anatómicas más grandes tienen entesis con grados de robusticidad más elevados. Esta situación es esperable ya que los individuos con mayor tamaño corporal requieren de mayor esfuerzo para moverse (Krantz, 1981). Lo interesante es que esta correlación ocurre para la mayoría de las entesis de los miembros inferiores (en los superiores solo se detectó para el pectoral mayor), lo que podría estar relacionado con el la influencia del peso corporal sobre el fémur y tibia. Ahora bien, los valores-z de los huesos largos también podrían considerarse como proxy del sexo de los individuos. De esta forma, la antedicha correlación entre tamaño corporal y grados de robusticidad elevados podría indicar que los individuos masculinos presentan mayores grados de robusticidad que los femeninos en los miembros inferiores y en el pectoral mayor. Esta posible asociación estaría explicada por diferencias hormonales entre ambos sexos así como por factores mecánicos. Los andrógenos promue- 
B. MAZZA/REV ARG ANTROP BIOL 22(1), 2020. doi:10.24215/18536387e010

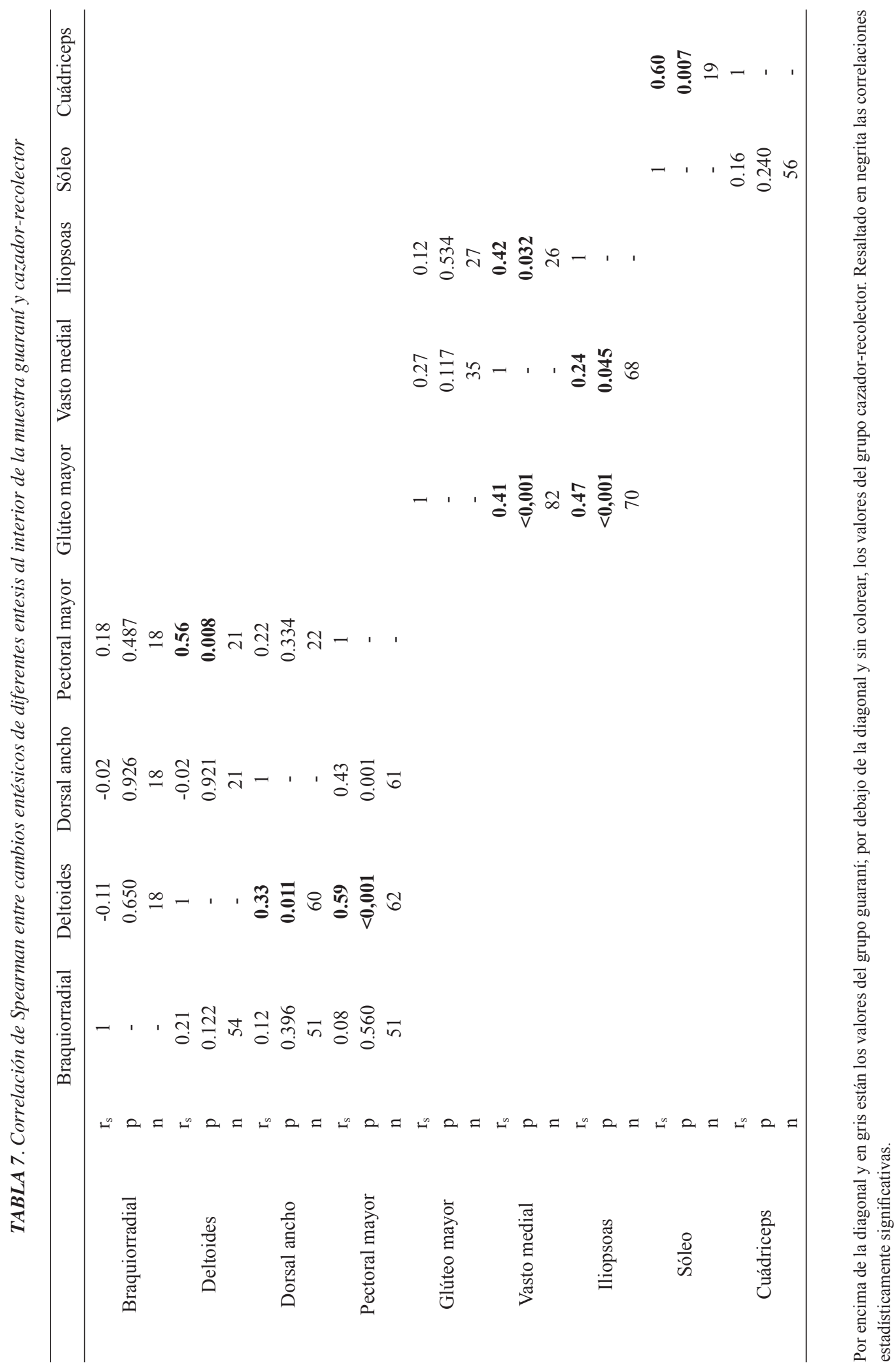


ven el crecimiento y la fuerza muscular y ósea (Notelovitz, 2002). Estas hormonas se encuentran en mayor proporción en los hombres, quienes al tener una mayor masa muscular presentan también mayor robusticidad en las entesis que las mujeres (Mazza, 2018; Niinimäki, 2012; Weiss, 2003). A su vez, el estrés mecánico estimula la acción de los osteocitos, los cuales inician la remodelación ósea (Notelovitz, 2002). Por lo tanto, los individuos masculinos tendrían mayores cambios entésicos en los miembros inferiores y en el pectoral mayor que los femeninos debido a la acción combinada de andrógenos y estrés mecánico. Teniendo esto en cuenta, llama la atención la ausencia de correlaciones significativas entre los valores-z y el resto de las entesis del húmero, lo cual se podría relacionar con los otros dos factores que explicarían la variabilidad de los cambios entésicos en el grupo guaraní; el estrés mecánico y el sesgo en la muestra. En este sentido, dicha falta de correlación podría estar indicando que los individuos de ambos sexos, o con diferentes tamaños corporales, estaban bajo presiones mecánicas similares derivadas de actividades cotidianas. Sin embargo, no puede descartarse que la ausencia de correlaciones significativas se deba a similitudes etarias entre los individuos analizados o a que la muestra no sea lo suficientemente representativa.

Los resultados de este trabajo también muestran que los cambios entésicos del grupo guaraní pueden agruparse en dos patrones según se consideren los miembros inferiores o superiores. En el caso de los miembros inferiores predominan grados de robusticidad leves; en cambio, la mayoría de las entesis del húmero muestran grados de robusticidad medios. Los miembros superiores están sujetos a una amplia variedad de actividades y, por ende, podrían haber sufrido mayor estrés mecánico o bien podrían ser más susceptibles a detectar diferentes presiones mecánicas que los miembros inferiores, limitados principalmente a actividades ambulatorias (Mazza, 2018; Niinimäki y Baiges Soto, 2013). Sin embargo, para poder sostener esta hipótesis, es necesario contar con un número mayor de muestras donde se pueda analizar la relación entre los cambios entésicos y la edad de los individuos; ya que, por ejemplo, dada la naturaleza secundaria de los entierros guaraníes, los húmeros podrían pertenecer a individuos de edades más avanzadas que aquellos de los miembros inferiores. Es decir que esta variabilidad podría estar relacionada con un sesgo en la muestra.

Las características de las entesis recientemente descriptas del grupo guaraní guardan similitudes con los cambios entésicos de los miembros inferiores del grupo c-r (con excepción del vasto medial) y diferencias en las entesis del húmero donde el grupo guaraní presenta mayores cambios entésicos que los c-r. En primer lugar, estos resultados podrían deberse a que la muestra del grupo guaraní estuviera conformada por una mayor proporción de individuos masculinos en comparación al del c-r. Sin embargo, cuando se realizó el MLG sin los femeninos del grupo c-r los resultados no se modificaron. Por lo tanto, una disparidad en la conformación sexual de las muestras no sería uno de los factores que explique las diferencias observadas. En cuanto a la edad, cabe la posibilidad de que la muestra del grupo guaraní tenga una mayor proporción de individuos de edades más avanzadas que los c-r, siendo la causa de las diferencias en el húmero y en el vasto medial. Lamentablemente, no se pueden hacer comparaciones como la recién descripta para el sexo porque solo unos pocos individuos adultos de la muestra c-r pudieron ser clasificados en rangos etarios (Mazza, 2018). Esto es un aspecto que deberá comprobarse a futuro. Otro factor es el tamaño corporal, el cual fue controlado en los MLG, y por lo tanto no interviene en los resultados. Por último, nos queda el estrés mecánico. La mayoría de las entesis de los miembros inferiores no muestran diferencias significativas entre ambos grupos, lo cual podría atribuirse a una homogeneidad en la morfología del terreno del sector continental e insular (Acosta, Henderson y Cunha, 2017; Salega y Fabra, 2017) o bien a que los miembros inferiores están involucrados en actividades cotidianas (mayormente de carácter ambulatorio) que no suelen diferir entre los individuos. Además, están adaptados a soportar cargas mecánicas, como ser la ejercida por el peso corporal (Niinimäki y Baiges Soto, 2013; Weiss, 2004). Por lo tanto, otros investigadores propusieron que sutiles variaciones en las actividades ambulatorias no tendrían un impacto significativo en los cambios entésicos de los miembros inferiores (Niinimäki y Baiges Soto, 2013). Sin embargo, los guaraníes presen- 
tan una mayor robusticidad en el vasto medial que los c-r que podría estar relacionada a diferentes modalidades de desplazamiento espacial. Al respecto, Loponte et al. (2011) sugieren que la población guaraní del Paraná inferior habría tenido rangos de acción más dilatados y redes de intercambio más largas, realizados a través de una mayor movilidad fluvial con canoas, que los c-r locales. El vasto medial no solo tiene un rol fundamental en todas las actividades ambulatorias (caminar, correr) sino también en actividades de remo (Mazzone, 1988; Vergara Amador y Román Chalarca, 2011). Por lo tanto, es posible que los guaraníes hayan tenido una exigencia mecánica mayor que los c-r, la cual propició los cambios entésicos en el vasto medial como repuesta adaptativa al estrés. Si esta diferencia entre ambos grupos se debiera a que la muestra guaraní contiene una cantidad mayor de individuos con edades superiores a los 40 años, sería esperable que también presenten mayores cambios entésicos para las otras entesis de los miembros inferiores.

Por otra parte, las diferencias en las entesis del húmero entre los c-r y guaraníes también podrían explicarse por el uso intensivo de canoas por parte de los guaraníes, su dependencia de la horticultura y distintos equipamientos tecnológicos en ambos grupos. Todas estas diferencias apuntan a que los guaraníes y c-r estuvieron bajo distintas presiones mecánicas. Este hecho podría explicar los contrastes en los patrones de correlación entre las entesis de los miembros superiores e inferiores al interior de la muestra de c-r y guaraní; ya que sus actividades habrían promovido la acción sinérgica de diferentes músculos.

Estas diferencias entre los guaraníes y c-r contradicen la tendencia general que marca una reducción en la robusticidad desde los c-r hacia sociedades productoras de alimentos (Henderson, 2013a; Larsen, 1995; Ruff, 1987). Es posible que esto se deba a que los guaraníes no eran sociedades que dependieran completamente de sus cultivos. Por el contrario, tenían una economía mixta, donde la caza y la pesca conformaban un aporte sustancial a la dieta al igual que la horticultura (Acosta et al., 2010a). Por lo tanto, es posible que este tipo de economía haya producido mayores presiones mecánicas en comparación al modo de vida c-r, sobrepasando el límite fi- siológico de las entesis, y por ende, produciendo cambios morfológicos en su superficie ósea. De hecho, los resultados de este trabajo confirman la hipótesis formulada por Henderson (2013a) que establece que los c-r tendrían menores cambios entésicos que los agricultores por estar mejor adaptados a su ambiente circundante, a pesar de que su análisis mostró el resultado contrario.

Por último, ambas poblaciones tienen trayectorias evolutivas diferentes, y por lo tanto, posiblemente diferencias genéticas, las cuales podrían influir en la morfología de sus entesis (Chen et al., 2007; Schlecht, 2012). Sin embargo, llama la atención que las diferencias entre ambos grupos ocurran principalmente en los miembros superiores. Es decir que si los patrones de los cambios entésicos de ambos grupos estuvieran influenciados por cuestiones genéticas, esperaríamos mayores diferencias en las entesis de los miembros inferiores.

\section{CONCLUSIONES}

En este trabajo hemos estudiado los cambios entésicos de individuos recuperados en sitios guaraníes del Delta inferior del río Paraná y sus diferencias con los cazadores-recolectores que habitaron el área hacia fines del Holoceno tardío. Al igual que sucede con el resto de las poblaciones donde se estudiaron los cambios entésicos, observamos que la variabilidad de los individuos guaraníes está posiblemente relacionada con diferencias en el tamaño corporal y con presiones mecánicas, aunque no se pueden descartar influencias del sexo y la edad, las cuales podrían explicar las diferencias observadas con la muestra de cazadores-recolectores. Estos patrones van en contra de la tendencia general en los cambios morfológicos postcraneales de sociedades c-r y productoras de alimentos, ya que los guaraníes presentan mayores cambios entésicos que los c-r. Aun necesitamos seguir avanzando en el estudio de diferencias morfológicas óseas entre ambas unidades arqueológicas. Para esto es necesario ampliar la muestra de restos óseos guaraníes con nuevas excavaciones en el área que nos permita estimar el sexo y edad de los individuos, a la vez que realizar nuevos estudios que comprendan el análisis de variables métricas y de propiedades geométricas transversales de las diáfisis. Esto nos permitirá complementar y poner a prueba 
los resultados de este trabajo cuyas muestras provienen, principalmente, de museos nacionales como resultado de excavaciones realizadas a principios del siglo XX.

\section{AGRADECIMIENTOS}

A Daniel Loponte por realizar una lectura crítica y comentarios de una versión de este manuscrito, a los encargados de las colecciones de bioarqueología del Museo de La Plata, Mariano del Papa y Andrés Di Bastiano. A Gustavo Barrientos por darme un lugar en su espacio de trabajo para analizar las colecciones en el Museo de Ciencias Naturales de La Plata.

\section{LITERATURA CITADA}

Acosta, A., Loponte, D., y Mucciolo, L. (2010a). Comparando estrategias de explotación faunística en el humedal del Paraná inferior: cazadores-recolectores versus horticultores amazónicos. En M. Gutiérrez, M. De Nigris, P. Fernández, M. Giardina, A. Gil, A. Izeta y H. Yacobaccio (Eds.). Zooarqueología a principios del siglo XXI. Aportes teóricos, metodológicos y casos de estudio (pp. 177-188). Buenos Aires, Argentina: El Espinillo.

Acosta, A., Loponte, D., y Mucciolo, L. (2010b). Uso del espacio y subsistencia de grupos horticultores amazónicos en el humedal del Paraná inferior. Arqueología Rosarina Hoy, 2, 35-55.

Acosta, A., y Ríos Román, V. (2013). Explotación prehispánica de palmeras por grupos cazadores-recolectores y horticultores del extremo sur de Sudamérica: el caso del humedal del Paraná inferior (Argentina). Pesquisas, Antropología, 70, 197-216.

Acosta, M., Henderson, C., y Cunha, E. (2017). The effect of terrain on entheseal changes in the lower limbs. International Journal of Osteoarchaeology, 27(5), 828838. doi: 10.1002/oa.2597

Alves Cardoso, F., y Henderson, C. (2010). Enthesopathy formation in the humerus: data from known age-at-death and known occupation skeletal collections. American Journal of Physical Anthropology, 141(4), 550-560. doi: 10.1002/ajpa.21171

Ambrosetti, J. (1895). Los cementerios prehistóricos del Alto Paraná (Misiones). Boletín del Instituto Geográico Argentino 16, 227-263.

Benjamin, M., Kumai, T., Milz, S., Boszczyk, B., Boszczyk, A., y Ralphs, J. R. (2002). The skeletal attachment of tendons-tendon 'entheses'. Comparative Biochemistry and Physiology, Part A(133), 931-945.

Bridges, P., Blitz, E., y Solano, M. (2000). Changes in long bone diaphyseal strength with horticultural intensification in west-central Illinois. American Journal of Physical Anthropology, 112, 217-238. doi:10.1002/ ajpa.21377

Buc, N., y Loponte, D. (2007). Bone tool types and microwear patterns: some examples from the Pampa Region, South America. En C. G. St-Pierre y R. B. Walker (Eds.).Bones as tools: current methods and interpretations in worked bone studies (pp. 143-157). Oxford, Reino Unido: BAR International Series 1622.
Buikstra, J., y Ubelaker, D. (1994). Standards for data collection from human skeletal remains. Arkansas, Estados Unidos: Arkansas Archaeological Survey.

Caggiano, M. A., Flores, O., Méndez, M., y Salceda, S. (1978). Nuevos aportes para el conocimiento antropológico del delta del Paraná. Relaciones de la Sociedad Argentina de Antropología, XII, 155-174.

Chapman, N. (1997). Evidence for Spanish influence on activity induced musculoskeletal stress markers at Pecos Pueblo. International Journal of Osteoarchaeology, 7, 497-506. doi: 10.1002/(SICI)10991212(199709/10)7:5<497::AID-OA394>3.0.CO;2-H

Chen, X., Macica, C., Nasiri, A., Judex, S., y Broadus, A. (2007). Mechanical regulation of PTHrP expression in entheses. Bone, 41(5), 752-759.

Chrisletta, P., y Spini, D. (2004). An introduction to generalized estimating equations and an application to assess selectivity effects in a longitudinal study on very old individuals. Journal of Educational and Behavioral Statistics, 29, 421-437.

Eshed, V., Gopher, A., Galili, E., y Hershkovitz, I. (2004). Musculoskeletal stress markers in natufian hunter-gatherers and neolithic farmers in the Levant: the upper limb. American Journal of Physical Anthropology, 123, 303-315. doi: 10.10

Frayer, D., y Wolpoff, M. (1985). Sexual dimorphism. Annual Review of Anthropology, 14, 429-473.

Henderson, C. (2013a). Subsistence strategy changes: the evidence of entheseal changes. Homo, 64(6), 491-508. doi: 10.1016/j.jchb.2013.08.002

Henderson, C. (2013b). Do diseases cause entheseal changes at fibrous entheses? International Journal of Paleopathology, 3(1), 64-69. doi: 10.1016/j.ijpp.2013.03.007

Henderson, C., Mariotti, V., Pany-Kucera, D., Villote, S., y Wilczak, C. (2013). Recording specific entheseal changes of fibrocartilaginous entheses: initial tests using the Coimbra method. International Journal of Osteoarchaeology, 23, 152-162. doi: 10.1002/oa.2287

Henderson, C., Mariotti, G., Pany-Kucera, D., Villote, S., y Wilczak, C. (2016). The new 'Coimbra method': a biologically appropriate method for recording specific features of fibrocartilaginous entheseal changes. International Journal of Osteoarchaeology, 26, 925-932. doi: 10.1002/oa.2477

IBM Corp. (2010). IBM SPSS Statistics for Windows, Version 19.0. Armonk, Estados Unidos: IBM Corp.

Jurmain, R., Alves Cardoso, F., Henderson, C., y Villote, S. (2012). Bioarchaeology's Holy Grail: The reconstruction of activity. En A. Grauer (Ed.). A Companion to Paleopathology (pp. 531-552). Chichester, Reino Unido: Blackwell.

Krantz, G. (1981). The process of human evolution. Cambridge, Reino Unido: Schenkman Publishing Company Inc.

Larsen, C. S. (1995). Biological changes in human populations with agriculture. Annual Review of Anthropology, 24, 185-213.

Loponte, D. (2008). Arqueología del humedal del Paraná inferior (Bajios Ribereños Meridionales). Buenos Aires, Argentina: Ediciones del Riel.

Loponte, D., y Acosta, A. (2003-2005). Nuevas perspectivas para la arqueología "guaraní" en el humedal del Paraná inferior y Río de la Plata. Cuadernos del Instituto $\mathrm{Na}$ cional de Antropología y Pensamiento Latinoamericano, 20, 179-197.

Loponte, D., y Acosta, A. (2013). La construcción de la unidad arqueológica guaraní en el extremo meridional de su distribución geográfica. Cuadernos del Instituto 
Nacional de Antropología y Pensamiento Latinoamericano, Series Especiales, 1(4), 193-235.

Loponte, D., y Acosta, A. (2016). Los contextos GoyaMalabrigo del noreste argentino. Cadernos do CEOM, 29(45), 125-187. doi: 10.22562/2016.45.06

Loponte, D., Acosta, A., Capparelli, I., y Pérez, M. (2011). La arqueología guaraní en el extremo meridional de la cuenca del Plata. En D. Loponte y A. Acosta (Eds.). Arqueología Tupiguarani (pp. 111-154). Buenos Aires, Argentina: Instituto Nacional de Antropología y Pensamiento Latinoamericano.

Loponte, D., Acosta, A., y Corriale, M. J. (2016). Isotopic trends in the diets of hunter-gatherers of the lower Paraná wetland, South America. Journal of Archaeological Science: Reports, 9, 259-274. doi: 10.1016/j.jasrep.2016.07.023

Loponte, D., Acosta, A., Mucciolo, L. (2012). Contribución a la arqueología del delta del Paraná: el nivel acerámico del sitio Isla Lechiguanas 1. Comechingonia, 16, 229-268.

Lothrop, S. (1932). Indians of the Parana delta. Annals of the New York Academy of Science, XXXIII, 77-232.

Mariotti, V., Facchini, F., y Belcastro, G. (2007). The study of entheses: proposal of a standardised scoring method for twenty-three entheses of the postcranial skeleton. Collegium Antropologicum 31, 291-313.

Mazza, B. (2010). Cerro Lutz: aproximaciones al estudio de las prácticas mortuorias de las sociedades cazadoras-recolectoras del humedal del Paraná inferior. La Zaranda de Ideas, 6, 91-116.

Mazza, B. (2015). Colecciones antiguas, datos nuevos: primeros resultados del análisis de las colecciones bioarqueológicas del humedal del Paraná inferior. Revista del Museo de Antropología, 8(1), 133-146.

Mazza, B. (2018). Entheseal changes among late Holocene hunter-gatherers from the southern extreme of La Plata basin (Argentina). Archaeological and Anthropological Sciences. doi: 10.1007/s12520-018-0638-2.

Mazza, B., Acosta, A., y Loponte, D. (2016). Nuevos Datos para las inhumaciones en urnas de sitios arqueológicos guaraníes del extremo meridional de la Cuenca del Plata. Revista Chilena de Antropología, 34, 81-96.

Mazza, B., y Loponte, D. (2012). Las prácticas mortuorias en el humedal del Paraná inferior. Arqueología Iberoamericana, 13, 3-21.

Mazzone, T. (1988). Kinesiology of the rowing stroke. NSCA Journal, 10(2), 4-11.

McCullagh, P., y Nelder, J. A. (1989). Generalized linear models. Londres, Reino Unido: Chapman and Hall.

Métraux, A. (1948). The Guaraní. En J. Steward (Ed.). Handbook of South American Indians (Vol. 3, pp. 69-94). Washington, Estados Unidos: Smithsonian Institute.

Milella, M., Belcastro, M., Zollikofer, C., y Mariotti, V. (2012). The effect of age, sex, and physical activity on entheseal morphology in a contemporary italian skeletal collection. American Journal of Physical Anthropology, 148, 379-388. doi: 10.1002/ajpa.22060.

Müller, L. M., y Mendonça de Souza, S. (2011). Enterramentos Guarani: problematizaçao e novos achados. En M. Carbonera y P. I. Schmitz (Eds.). Antes do oeste catarinense. Arqueologia dos povos indigenas (pp. $167-$ 218). Chapecó, Brasil: Argos.

Neves, W., Bernardo, D., Okumura, M., Ferreira de Almeida, T., y Strauss, A. (2011). Origem e dispersão dos Tupiguarani: o que diz a morfologia craniana? Boletim do Museu Paraense Emílio Goeldi. Ciências Humanas, 6(1), $95-$ 122.

Niinimäki, S. (2012). The relationship between musculoske- letal stress markers and biomechanical properties of the humeral diaphysis. American Journal of Physical Anthropology, 147(4), 618-628. doi: 10.1002/ajpa.22023.

Niinimäki, S., y Baiges Sotos, L. (2013). The relationship between intensity of physical activity and entheseal changes on the lower limb. International Journal of Osteoarchaeology, 23(2), 221-228. doi: 10.1002/oa.2295

Nikita, E. (2017). Osteoarchaeology. A guide to the macroscopic study of human skeletal remains. Londres, Reino Unido: Elsevier.

Noelli, F. (2008). The Tupi expansion. En H. Silverman y W. H. Isbell (Eds.). The handbook of South American archaeology (pp. 659-670). New York, Estados Unidos: Springer.

Notelovitz, M. (2002). Androgen effects on bone and muscle. Fertility and Sterility, 77(4), S34-S41.

Outes, F. (1918). Nuevos rastros de la cultura Guaraní en la cuenca del Paraná inferior. Anales de la Sociedad Cientíica Argentina, LXXXII, 153-182.

Pérez, M., y Cañardo, L. (2004). Producción y uso de cerámica en el norte de la provincia de Buenos Aires. En G. Martínez, M. Gutiérrez, R. Curtoni, M. Berón y P. Madrid (Eds.). Aproximaciones contemporáneas a la Arqueología Pampeana. Perspectivas teóricas, metodológicas, analíticas y casos de estudio (pp. 335-347). Olavarría, Argentina: UNCPBA.

Pérez, M., Silvestre, R., y Buc, N. (2019). Tecnología de grupos guaraníes en las cuencas alta y baja de los ríos Paraná y Uruguay. Revista de Antropología del Museo de Entre Ríos, 4(2), 41-65.

Politis, G., Bastourre, L., Di Prado, V., Bonomo, M., Moreira, G., y Matarrese, A. (2017). El Túmulo II del Brazo Largo. Aportes para la arqueología del Delta Inferior del río Paraná. Revista del Museo de Antropología, 10, 71-88.

Prous, A. (2011). Estudios sobre los portadores de la cerámica tupiguarani en Brasil: proto-Tupi, proto-Guarani y otros. En D. Loponte y A. Acosta (Eds.). Arqueología Tupiguaraní (pp. 23-110). Buenos Aires, Argentina: Instituto Nacional de Antropología y Pensamiento Latinoamericano.

Resnick, D., y Niwayama, G. (1983). Entheses and enthesopathy. Radiology, 146, 1-9.

Rodríguez, J. (2008). Arqueología de humedales en la provincia de Corrientes (Argentina). En D. Loponte y A. Acosta (Eds.). Entre la tierra y el agua, Arqueología de humedales del Este de Sudamérica (pp. 165-190). Buenos Aires, Argentina: Editorial Los Argonautas.

Ruff, C. (1987). Sexual dimorphism in human lower limb bone structure: relationship to subsistence strategy and sexual division of labor. Journal of Human Evolution, 16, 391-416.

Sacur Silvestre, R., Buc, N., Acosta, A., \& Loponte, D. (2013). Estrategias de captura de presas y sistemas de armas de los cazadores-recolectores que habitaron el humedal del Paraná inferior: una aproximación experimental y arqueológica. Comechingonia, 17, 22-57.

Salega, S., y Fabra, M. (2017). Variaciones del terreno y cambios entesiales en poblaciones prehispánicas de Córdoba (Argentina). Revista del Museo de Antropología, 10(2), 131-136.

Scheuer, L., y Black, S. (2000). Developmental Juvenile Osteology. San Diego,Estados Unidos: Elsevier.

Schlecht, S. (2012). Understanding entheses: bridging the gap between clinical and anthropological perspectives. The Anatomical Record: Advances in Integrative Anatomy and Evolutionary Biology, 295(8), 1239-1251. doi: 10.1002/ar.22516

Shuler, K. A., Zeng, P., y Danforth, M. E. (2012). Upper limb entheseal change with the transition to agriculture 
in the Southeastern United States: a view from Moundville and the central Tombigbee River valley. Homo, 63(6), 413-434. doi: 10.1016/j.jchb.2012.09.002.

Torres, L. M. (1911). Los primitivos habitantes del delta del Paraná. Buenos Aires, Argentina: Coni Hermanos.

Vergara Amador, E., y Román Chalarca, M. (2011). Descripción anatómica del músculo vasto medial. ¿Existe realmente el músculo vasto medial oblicuo? Salud Uninorte, 27(1), 73-84.

Vignati, M. (1941). Censo óseo de paquetes funerarios de origen guaraní. Revista del Museo de La Plata (Nueva Serie), Sección Antropología, 9(II), 1-11.

Vignati, M. (1960). El indigenado en la pcia. de Buenos Aires. Anales de la Comisión de Investigaciones Cientificas, I, 95-182.

Villotte, S., Assis, S., Cardoso, F. A,. Henderson, C., Mariotti, V., Milella, et al. (2016). In search of consensus: terminology for entheseal changes (EC). International Journal of Paleopathology, 13, 49-55. doi: 10.1016/j. ijpp.2016.01.003.

Weiss, E. (2003). Understanding muscle markers: aggregation and construct validity. American Journal of Physical Anthropology, 121, 230-240. doi: 10.1002/ ajpa. 10226

Weiss, E. (2004). Understanding muscle markers: lower limbs. American Journal of Physical Anthropology, 125(3), 232-238. doi: 10.1002/ajpa.10397.

Weiss, E., Corona, L., y Schultz, B. (2012). Sex differences in musculoskeletal stress markers: problems with activity pattern reconstructions. International Journal of Osteoarchaeology, 22, 70-80. doi: 10.1002/oa.1183

White, T. (1953) A method of calculating the dietary percentage of various food animals utilized by aboriginal people. American Antiquity, 18, 396-398. 